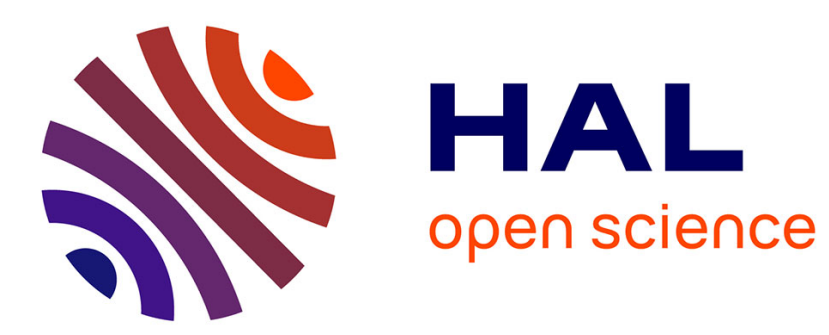

\title{
Robust goal-oriented error estimation based on the constitutive relation error for stochastic problems
}

Ludovic Chamoin, Éric Florentin, Sylvain Pavot, Vincent Visseq

\section{To cite this version:}

Ludovic Chamoin, Éric Florentin, Sylvain Pavot, Vincent Visseq. Robust goal-oriented error estimation based on the constitutive relation error for stochastic problems. Computers \& Structures, 2012, 106-107, pp.189-195. 10.1016/j.compstruc.2012.05.002 . hal-00776138

\section{HAL Id: hal-00776138 \\ https://hal.science/hal-00776138}

Submitted on 15 Jan 2013

HAL is a multi-disciplinary open access archive for the deposit and dissemination of scientific research documents, whether they are published or not. The documents may come from teaching and research institutions in France or abroad, or from public or private research centers.
L'archive ouverte pluridisciplinaire HAL, est destinée au dépôt et à la diffusion de documents scientifiques de niveau recherche, publiés ou non, émanant des établissements d'enseignement et de recherche français ou étrangers, des laboratoires publics ou privés. 


\title{
Robust goal-oriented error estimation based on the
}

\section{constitutive relation error for stochastic problems}

\author{
Ludovic Chamoin $^{\mathrm{a}}$, Eric Florentin ${ }^{\mathrm{a}, *}$, Sylvain Pavot ${ }^{\mathrm{a}}$, Vincent Visseq ${ }^{\mathrm{b}}$ \\ a LMT-Cachan (ENS Cachan/CNRS/Paris 6 Univ./PRES UniverSud Paris) \\ 61 Avenue du Président Wilson, 94235 CACHAN Cedex, France \\ b Laboratoire de Mécanique et Génie Civil (LMGC), UMR5508 CNRS / Université Montpellier 2 \\ CC048, Place E. Bataillon, 34095 Montpellier Cedex 5 - France
}

\begin{abstract}
In this paper, we aim at extending to stochastic models a general and robust goal-oriented error estimation method presented in previous works. This method, which is based on the constitutive relation error and classical extraction techniques, enables to obtain strict bounds on quantities of interest. In the stochastic framework, several aspects are revisited in the current paper:(i) the construction of admissible fields, which is a pillar of the constitutive relation error; (ii) the error bounding itself; (iii) the splitting of error sources that may enable to drive adaptive procedures effectively. Performances of the proposed approach are illustrated on two-dimensional applications.
\end{abstract}

Key words: model verification, stochastic models, goal-oriented error estimation, strict bounds 


\section{Introduction}

In the design of engineering components and structures, critical decisions are being more and more based on the results coming from finite element analyses. Therefore, in order to develop confidence in such decisions, controlling the quality of numerical simulations has become a vital issue in both research and industry. This research topic, referred to as model verification, has been extensively studied for more than thirty years and has led to the emergence of powerful methods, particularly as regards the assessment of the global discretization error (see $[1,18]$ for an overview). More recently, research has focused on goaloriented error estimation, i.e. the estimation of the error on specific outputs of interest which may be relevant for design purposes. Several techniques have been proposed for goal-oriented error estimation, and particularly for linear problems $[28,7,30,34,38,10]$. However, only few of these actually lead to strict error bounds.

A general framework was recently introduced for robust goal-oriented error estimation; it has the advantage to be valid for a large class of mechanical problems [17,22]. This framework, based on the concept of constitutive relation error, in association with extraction techniques (that require the solution of an adjoint problem), enables the calculation of strict and accurate bounds on the local error. The method has been recently and successfully applied to various problems such as fracture mechanics tackled with XFEM [27], (visco-)elasticity [5], transient viscodynamics [21], or (visco-)plasticity. In [6,22], a non-intrusive approach was also added to this framework in order to solve the adjoint problem in an optimal manner, which enables in particular to consider pointwise quantities of interest in time and space. This powerful approach consists in a local enrichment of the adjoint solution, using pre-computed generalized Green's functions, in order to catch effectively and at reasonable cost the locally irregular aspects of this solution.

\footnotetext{
*. Corresponding author

Email addresses: chamoin@lmt.ens-cachan.fr (Ludovic Chamoin), florentin@lmt.ens-cachan.fr (Eric Florentin), pavot@lmt.ens-cachan.fr (Sylvain Pavot), visse@lmt.ens-cachan.fr (Vincent Visseq).
} 
During the last decade, with the fast increase of computing resources, complex models involving stochastic parameters have been introduced in the computational mechanics community. Such models, which are more and more employed and simulated nowadays $[13,36,24,9,37,2]$, enable to represent lacks of knowledge in the modeling process as well as intrinsic physical randomness. As regards the verification of stochastic models, most of the works are devoted to global error estimation (see $[14,16]$ for instance). For goal-oriented error estimation, the proposed methods $[26,19,12]$ apply to a specific set of quantities of interest and do not yield strict error bounds (only error indicators obtained through heuristic arguments).

In this work, we aim at extending the previously introduced general goal-oriented error estimation method to stochastic mechanical models. In order to do so, a first key point to consider is the construction, in a stochastic sense, of an admissible solution which is required to apply the constitutive relation error ; this point was first addressed in [16]. Furthermore, we need to extend the bounding result obtained for the local error. A third point should deal with the splitting of error sources (i.e. error contributions due to discretizations in space and stochastic dimensions in our case), and assessment of these contributions in order to drive adaptive algorithms effectively, if necessary [11].

Consequently, the paper is structured as follows : after this introduction, Section 2 describes the stochastic reference problem we consider throughout the paper, and gives details about the computation of an associated approximate solution; Section 3 recalls, for the stochastic framework, the main features of the constitutive relation error and the construction of an admissible solution; Section 4 introduces the stochastic version of the goal-oriented error estimation method we use, as well as the procedure employed to estimate contributions of various error sources ; numerical results are presented in Section 5 ; eventually, conclusions and prospects are drawn in Section 6 . 


\section{Reference problem and notations}

\subsection{The stochastic reference problem}

We consider an open bounded domain $\Omega \in \mathbb{R}^{d}$, with $d$ the spatial dimension, representing a mechanical structure in a given environment (see Figure 1). This structure, whose boundary is denoted $\partial \Omega$, is subjected to a prescribed external loading represented by a displacement field $\mathbf{u}_{d} \in\left[L^{2}(\partial \Omega)\right]^{d}$ over $\partial_{1} \Omega \subset \partial \Omega$ such that $\partial_{1} \Omega \neq \varnothing$, a traction force $\mathbf{F}_{d} \in\left[L^{2}(\partial \Omega)\right]^{d}$ over $\partial_{2} \Omega\left(\right.$ with $\partial_{1} \Omega \cap \partial_{2} \Omega=\varnothing$ and $\overline{\partial_{1} \Omega \cup \partial_{2} \Omega}=\partial \Omega$ ), and a body force field $\mathbf{f}_{d} \in\left[L^{2}(\Omega)\right]^{d}$ within $\Omega$. This loading is assumed to be deterministic, even though the methodology presented in the paper could be easily extended to stochastic loadings.

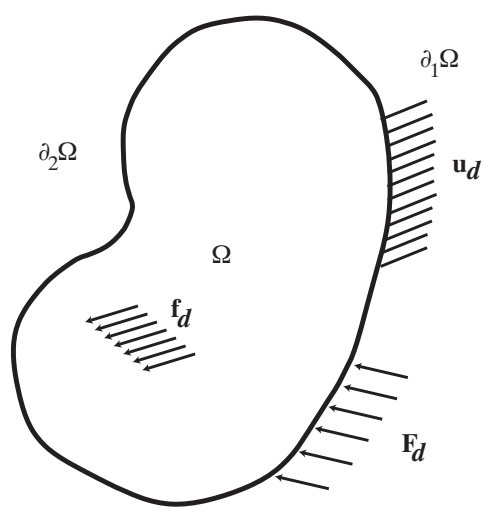

Figure 1. The structure considered and its environment.

The material that constitutes $\Omega$ is assumed to be linear and elastic, and $\boldsymbol{K}$ denotes the corresponding Hooke tensor. Moreover, we consider random fluctuating material parameters so that the Hooke tensor is modeled by a random field $\boldsymbol{K}(\mathbf{x}, \theta) \in\left[L^{2}\left(\Theta, C^{0}(\Omega)\right)\right]^{d^{4}} ;(\Theta, \mathcal{F}, P)$ is a complete probability space defined in the Kolmogorov sense [15], with $\Theta$ the set of possible outcomes, $\mathcal{F}$ a $\sigma$-algebra of events (subsets of $\Theta$ ), and $P: \mathcal{F} \rightarrow[0,1]$ a probability measure. We assume that the field $\boldsymbol{K}(\mathbf{x}, \theta)$ is bounded and uniformly coercive, that is $\left.\exists\left(K_{\min }, K_{\max }\right) \in\right] 0,+\infty\left[^{2}\right.$ such that :

$$
0<K_{\min } \leq|\boldsymbol{K}(\mathbf{x}, \theta)| \leq K_{\max } \quad \forall \mathbf{x} \in \Omega, \text { almost surely }
$$


Remark 1 Following the Karhunen-Loeve expansion [23], the stochastic description of $\boldsymbol{K}$ will be limited to a finite number of $M$ uncorrelated stochastic variables $\xi_{k}(\theta): \Theta \rightarrow \mathbb{R}$ such that :

$$
\boldsymbol{K}(\mathbf{x}, \theta) \approx \overline{\boldsymbol{K}}(\mathbf{x})+\sum_{k=1}^{M} \sqrt{\lambda_{k}} \xi_{k}(\theta) \boldsymbol{Z}_{k}(\mathbf{x})
$$

where $\overline{\boldsymbol{K}}=\int_{\Theta} \boldsymbol{K} d P$ is the mean value of $\boldsymbol{K}$, whereas $\left\{\boldsymbol{Z}_{k}, \lambda_{k}\right\}$ are eigenvector/eigenvalue pairs of the covariance operator. This truncation at order $M$ provides for an approximation of $\boldsymbol{K}$.

We equip the space $(\Theta, \mathcal{F}, P)$ with an $L^{2}$-inner product on probability measures, defined as :

$$
\langle\alpha, \beta\rangle \equiv \int_{\Theta} \alpha(\theta) \beta(\theta) d P(\theta)
$$

where $(\alpha, \beta)$ is a couple of random variables and $d P$ is the probability measure of $\theta$. We also define the following norms on $\Omega \times \Theta$ :

$$
\begin{aligned}
& \|\| \|_{\boldsymbol{K}}=\left(\mathbb{E}\left[\int \operatorname{Tr}[\mathbb{E}(\bullet) K \varepsilon(\bullet)] \mathrm{d} \Omega\right]\right)^{1 / 2}=\left(\mathbb{E}\left[\|\bullet\|_{\boldsymbol{K}}^{2}\right]\right)^{1 / 2} \\
& \|\| \bullet \|_{\boldsymbol{K}^{-1}}=\left(\mathbb{E}\left[\int \operatorname{Tr}\left[\bullet \boldsymbol{K}^{-1} \bullet\right] \mathrm{d} \Omega\right]\right)^{1 / 2}=\left(\mathbb{E}\left[\|\bullet\|_{\boldsymbol{K}^{-1}}^{2}\right]\right)^{1 / 2}
\end{aligned}
$$

where $\mathbb{E}(\bullet)=\int_{\Theta} \bullet d P$ is the mathematical expectation of $\bullet$.

Assuming an isothermal state with small perturbations, the quasi-static problem consists of finding the displacement-stress pair $(\mathbf{u}(\mathbf{x}, \theta), \sigma(\mathbf{x}, \theta))$ which verifies :

- the kinematic compatibility equations :

$$
\mathbf{u} \in \mathcal{U} \quad ; \quad \mathbf{u}_{\mid \partial_{1} \Omega}=\mathbf{u}_{d} \quad \text { almost surely }
$$

- the equilibrium equations :

$$
\sigma \in \mathcal{S} \quad ; \quad \mathbb{E}\left[\int_{\Omega} \operatorname{Tr}\left[\sigma \notin\left(\mathbf{u}^{*}\right)\right] \mathrm{d} \Omega-\int_{\Omega} \mathbf{f}_{d} \cdot \mathbf{u}^{*} \mathrm{~d} \Omega-\int_{\partial_{2} \Omega} \mathbf{F}_{d} \cdot \mathbf{u}^{*} \mathrm{~d} S\right]=0 \quad \forall \mathbf{u}^{*} \in \mathcal{U}_{0}
$$

- the constitutive relation :

$$
\sigma=\boldsymbol{K} \notin(\mathbf{u})
$$

where $\mathcal{U}=\left[L^{2}\left(\Theta, H^{1}(\Omega)\right)\right]^{d}, \mathcal{S}=\left\{\tau ; \tau=\tau^{T}, \tau \in\left[L^{2}\left(\Theta, L^{2}(\Omega)\right)\right]^{d^{2}}\right\}$, and $\mathcal{U}_{0}$ is the vectorial space associated with $\mathcal{U} \cdot \varepsilon(\bullet)=\frac{1}{2}\left[\mathbb{G r a d} \bullet+\mathbb{G r a d}^{T} \bullet\right]$ is the linearized strain tensor. 


\subsection{Discretization errors}

The exact solution of problem $(5-7)$ is denoted $\left(\mathbf{u}_{e x}, \sigma_{e x}\right)$. In practice, it is approximated using a stochastic finite element method (SFEM) [39]. In the space dimension, we use a discretization of $\Omega$, based on mesh $\mathcal{M}_{h}$. In the stochastic dimension, the discretization used for $\Theta$ is based on a grid $\mathcal{M}_{m}$. Two families of techniques exist :

- non-intrusive techniques, such as Monte Carlo methods or regression methods, in which a set of events is drawn to compute realizations in a deterministic way;

- intrusive techniques, such as the (generalized) Polynomial Chaos associated with the stochastic finite element method, which search an approximate solution in a finite dimension space.

In both cases, polynomial chaos is often used for $\mathcal{M}_{m}$. This space is defined from a polynomial basis $\left\{\Psi_{i}\right\}_{i=1}^{L}$ of variables $\left\{\xi_{k}(\theta)\right\}_{k=1}^{M}$. Namely, elements of the basis are defined as $\Psi_{i}\left(\left\{\xi_{k}(\theta)\right\}_{k=1}^{M}\right)=$ $\prod_{k=1}^{M} H_{k, i}\left(\xi_{k}\right)$, where $H_{k, i}\left(\xi_{k}\right)$ are orthonormal polynomials with respect to the inner-product defined in (3). A review on these various possible techniques that yield approximate stochastic solutions can be found in [4]. In the following, and without loss of generality, we consider a non-intrusive technique based on interpolation, over the stochastic domain, of a given number of computed realizations. More precisely, $L$ deterministic simulations are performed and lead to displacement fields $\mathbf{u}_{h}^{i}(\mathbf{x})(i=1, \ldots, L)$. The stochastic field $\mathbf{u}_{h, m}(\mathbf{x}, \theta)$ is then obtained after interpolation using shape functions $\Psi_{i}(i=1, \ldots, L) ; \mathbf{u}_{h, m}$ then reads :

$$
\mathbf{u}_{h, m}(\mathbf{x}, \theta)=\sum_{i=1}^{L} \mathbf{u}_{h}^{i}(\mathbf{x}) \cdot \Psi_{i}\left(\left\{\xi_{k}(\theta)\right\}_{k=1}^{M}\right)=\sum_{i=1}^{L} \mathbf{u}_{h}^{i}(\mathbf{x}) . \Psi_{i}(\theta)
$$

The approximated solution is denoted $\left(\mathbf{u}_{h, m}, \sigma_{h, m}\right)$, where $\sigma_{h, m}=\boldsymbol{K}_{\mathbb{E}}\left(\mathbf{u}_{h, m}\right)$. Subscript $h(\operatorname{resp} . m)$ denotes the discretization in the space (resp. stochastic) dimension related to mesh $\mathcal{M}_{h}$ (resp. grid $\mathcal{M}_{m}$ ). Using then the energetic norm associated to operator $\boldsymbol{K}$, we define a measure of the global discretization 
error :

$$
\mathcal{E}_{g l o b}=|| \mathbf{u}_{e x}-\mathbf{u}_{h, m} \mid \|_{K}
$$

We can also define the discretization error on a quantity of interest $I(\mathbf{u})$ representing a specific feature of the global solution $\mathbf{u}$ :

$$
\mathcal{E}_{l o c}=I\left(\mathbf{u}_{e x}\right)-I\left(\mathbf{u}_{h, m}\right)=I_{e x}-I_{h, m}
$$

Such a quantity of interest could be the mean of a component of the displacement or stress on a given zone.

\section{Constitutive relation error}

\subsection{Definition and properties}

We first introduce the notion of admissibility for a displacement-stress pair. A solution $(\hat{\mathbf{u}}, \hat{\sigma}) \in \mathcal{U} \times \mathcal{S}$ is said admissible if $\hat{\mathbf{u}}$ verifies (5) and $\hat{\sigma}$ verifies (6). We will show in Section 3.2 that such a solution can be obtained as a post-processing of $\left(\mathbf{u}_{h, m}, \sigma_{h, m}\right)$.

We then define, for an admissible couple $(\hat{\mathbf{u}}, \hat{\sigma})$, the constitutive relation error in a stochastic sense :

$$
e_{\text {cre }}(\hat{\mathbf{u}}, \hat{\sigma})=\left\|\mid \hat{\sigma}-K_{\mathscr{E}}(\hat{\mathbf{u}})\right\| \|_{\boldsymbol{K}^{-1}} \geq 0
$$

This is a straightforward generalization of the classical constitutive relation error given for deterministic models [18] :

$$
e_{c r e, s p a}(\hat{\mathbf{u}}, \hat{\sigma})=\left\|\hat{\sigma}-\boldsymbol{K}_{\varepsilon}(\hat{\mathbf{u}})\right\|_{\boldsymbol{K}^{-1}}
$$

It is also easy to show that properties of this latter constitutive relation error (see [18]) extend to the stochastic formulation :

$$
\begin{gathered}
e_{c r e}(\hat{\mathbf{u}}, \hat{\sigma})=0 \Longleftrightarrow(\hat{\mathbf{u}}, \hat{\sigma})=\left(\mathbf{u}_{e x}, \sigma_{e x}\right) \quad \text { almost surely } \\
e_{c r e}^{2}(\hat{\mathbf{u}}, \hat{\sigma})=\left.\left\|\mathbf{u}_{e x}-\hat{\mathbf{u}}\right\|\right|_{\boldsymbol{K}} ^{2}+\left\||| \sigma_{e x}-\hat{\sigma}\right\|_{\boldsymbol{K}^{-1}}^{2}
\end{gathered}
$$




$$
e_{c r e}^{2}(\hat{\mathbf{u}}, \hat{\sigma})=4\|\| \sigma_{e x}-\hat{\sigma}^{*} \|_{K^{-1}}^{2}
$$

with $\hat{\sigma}^{*}=\frac{1}{2}[\hat{\sigma}+\boldsymbol{K} \notin(\hat{\mathbf{u}})]$

\subsection{Computation of admissible fields}

An admissible solution, denoted $\left(\hat{\mathbf{u}}_{h, m}, \hat{\sigma}_{h, m}\right)$ in the following, is computed from the approximate solution $\left(\mathbf{u}_{h, m}, \sigma_{h, m}\right)$ at hand. On the one hand, as regards the kinematically admissible displacement field $\hat{\mathbf{u}}_{h, m}$, we merely choose $\hat{\mathbf{u}}_{h, m}=\mathbf{u}_{h, m}$ even though other choices would be possible. On the other hand, the computation of a statically admissible stress field $\hat{\sigma}_{h, m}$ is a technical point of the method. It can be performed using various techniques $[18,8,20,25,29,33]$; here, we use the technique recently introduced in [20] which constitutes a good compromise between quality and computational cost [31,32]. The practical construction of $\hat{\sigma}_{h, m}$ from $\sigma_{h, m}$ is detailed below.

- Direct construction : not admissible

The finite element stress field is generally post-treated as :

$$
\sigma_{h, m}(\mathbf{x}, \theta)=\sum_{i=1}^{L} \sigma_{h}^{i}(\mathbf{x}) . \Psi_{i}(\theta)
$$

Starting from components $\sigma_{h, m}^{i}(\mathbf{x})$, it is possible to construct the associated admissible stress fields $\hat{\sigma}_{h, m}^{i}(\mathbf{x})$ using directly techniques developed in the deterministic framework (see [31,32] for more details).

$$
\bar{\sigma}_{h, m}(\mathbf{x}, \theta)=\sum_{i=1}^{L} \hat{\sigma}_{h, m}^{i}(\mathbf{x}) \cdot \Psi_{i}(\theta)
$$

The problem is that $\bar{\sigma}_{h, m}(\mathbf{x}, \theta)$ is not admissible in the general case, as it does not respect (6), i.e. the equilibrium equations over the whole space $\Theta$.

- Definition of $\mathcal{M}_{m}^{\prime}$ : 
To avoid the previous problem, and enable a systematic construction of admissible field $\hat{\sigma}_{h, m}(\mathbf{x}, \theta)$, we introduce a dedicated basis. Generally, $\mathbf{f}_{d}$ and $\mathbf{F}_{d}$ are chosen linear with random variables : we introduce here a piecewise linear grid . If other choices are made for $\mathbf{f}_{d}$ or $\mathbf{F}_{d}$, a compatible basis can then be chosen.

We introduce the grid $\mathcal{M}_{m}^{\prime}$ based on the same nodes as $\mathcal{M}_{m}$ but using multi-linear shape functions $\left\{\chi_{i}\right\}_{i=1}^{L}$. The $L$ multilinear shape functions of the $M$ random variables are defined by $: \chi_{i}\left(\left\{\xi_{k}(\theta)\right\}_{k=1}^{M}\right)=$ $\prod_{k=1}^{M} N_{k, i}\left(\xi_{k}\right)$, where $N_{k, i}\left(\xi_{k}\right)$ are the classical finite element unidimensional shape function relative to $\xi_{k}$.

We denote $\sigma_{h, m}^{P}$ the representation of $\sigma_{h, m}$ defined on $\mathcal{M}_{h}^{\prime}$ :

$$
\sigma_{h, m}^{P}(\mathbf{x}, \theta)=\sum_{i=1}^{L} \sigma_{h}^{\chi_{i}}(\mathbf{x}) \cdot \chi_{i}\left(\left\{\xi_{k}(\theta)\right\}_{k=1}^{M}\right)=\sum_{i=1}^{L} \sigma_{h}^{\chi_{i}}(\mathbf{x}) \cdot \chi_{i}(\theta)
$$

where $\sigma_{h, m}^{\chi_{i}}$ are components of the stress $\sigma_{h, m}^{P}$ on $\left\{\chi_{i}\right\}$.

Then components $\hat{\sigma}_{h}^{N i}(\mathbf{x})$ of the stress $\hat{\sigma}_{h, m}(\mathbf{x}, \theta)$ in the basis $\left\{\chi_{i}\right\}$ can be computed directly using the different techniques developed in deterministic framework from components $\sigma_{h}^{\chi_{i}}(\mathbf{x})[31,32]$.

$$
\hat{\sigma}_{h, m}(\mathbf{x}, \theta)=\sum_{i=1}^{L} \hat{\sigma}_{h}^{\chi_{i}}(\mathbf{x}) \cdot \chi_{i}(\theta)
$$

The introduction of the basis $\left\{\chi_{i}\right\}$ is done to ensure the admissibility of $\hat{\sigma}_{h, m}$. Indeed, as far as $\chi_{i}\left(\left\{\xi_{k}(\theta)\right\}_{k=1}^{M}\right)$ is a multi-linear function of the $M$ variables $\left\{\xi_{k}(\theta)\right\}_{k=1}^{M}$, any linear combination of admissible stress fields $\hat{\sigma}_{h}^{\chi_{i}}(\mathbf{x})$ (by deterministic construction) will remain admissible. The only assumption to make is that the loading remains linear with random variables $\left\{\xi_{k}(\theta)\right\}_{k=1}^{M}$ (which is not a strong assumption ....). 


\section{Goal-oriented error estimation}

\subsection{Adjoint problem}

Assuming it is linear with respect to $\mathbf{u}$, the quantity of interest $I$ is first written under the global form :

$$
I=\int_{\Theta} \int_{\Omega}\left\{\operatorname{Tr}\left[\tilde{\sigma}_{\Sigma} \mathscr{E}(\mathbf{u})\right]+\tilde{\mathbf{f}}_{\Sigma} \cdot \mathbf{u}\right\} \mathrm{d} \Omega d P
$$

where stress field $\tilde{\sigma}_{\Sigma}(\mathbf{x}, \theta)$ and body force field $\tilde{\mathbf{f}}_{\Sigma}(\mathbf{x}, \theta)$, which may be explicitly or implicitly given, are extractors defined on $\Omega \times \Theta$.

Using the optimal control approach proposed in [3], we define the adjoint problem related to $I$; it consists of finding the displacement-stress pair $(\tilde{\mathbf{u}}(\mathbf{x}, \theta), \tilde{\sigma}(\mathbf{x}, \theta))$ which verifies :

- the kinematic compatibility equations :

$$
\tilde{\mathbf{u}} \in \mathcal{U} \quad ; \quad \tilde{\mathbf{u}}_{\mid \partial_{1} \Omega}=\mathbf{0} \quad \text { almost surely }
$$

- the equilibrium equations :

$$
\tilde{\sigma} \in \mathcal{S} \quad ; \quad \mathbb{E}\left[\int_{\Omega} \operatorname{Tr}\left[\left(\tilde{\sigma}-\tilde{\sigma}_{\Sigma}\right) \mathbb{E}\left(\mathbf{u}^{*}\right)\right] \mathrm{d} \Omega-\int_{\Omega} \tilde{\mathbf{f}}_{\Sigma} \cdot \mathbf{u}^{*} \mathrm{~d} \Omega\right]=0 \quad \forall \mathbf{u}^{*} \in \mathcal{U}_{0}
$$

- the constitutive relation :

$$
\tilde{\sigma}=K_{\notin}(\tilde{\mathbf{u}})
$$

As for the primal problem, we compute an approximate displacement-stress pair $\left(\tilde{\mathbf{u}}_{h, m}(\mathbf{x}, \theta), \tilde{\sigma}_{h, m}(\mathbf{x}, \theta)\right)$ using the same mesh $\mathcal{M}_{h}$ and grid $\mathcal{M}_{m}$.

We also derive an admissible displacement-stress pair $\left(\hat{\tilde{\mathbf{u}}}_{h, m}(\mathbf{x}, \theta), \hat{\tilde{\sigma}}_{h, m}(\mathbf{x}, \theta)\right)$ using the same techniques as for the primal problem. 


\subsection{Error bounding}

From quantities previously computed for primal and adjoint problems, we obtain the fundamental relation :

$$
\mathcal{E}_{l o c}=I_{e x}-I_{h, m}=\mathbb{E}\left[\int_{\Omega} \operatorname{Tr}\left[\left(\hat{\tilde{\sigma}}_{h, m}-\boldsymbol{K} \notin\left(\hat{\tilde{\mathbf{u}}}_{h, m}\right)\right) \boldsymbol{K}^{-1}\left(\sigma_{e x}-\hat{\sigma}_{h, m}\right)\right] \mathrm{d} \Omega\right]
$$

This result, for which proof can be found in $[17,22]$, shows that local error $\mathcal{E}_{l o c}$ can be represented from global solutions of both reference and adjoint problems.

From (24), and using the Cauchy-Schwarz inequality, we eventually obtain the guaranteed upper bound $\hat{\mathcal{E}}_{\text {loc }}$ on the local error $\mathcal{E}_{\text {loc }}$ :

$$
\left|\mathcal{E}_{l o c}\right| \leq \hat{\mathcal{E}}_{l o c}
$$

with :

$$
\hat{\mathcal{E}}_{l o c}=e_{c r e}(\hat{\mathbf{u}}, \hat{\sigma}) \cdot e_{c r e}(\hat{\tilde{\mathbf{u}}}, \hat{\tilde{\sigma}})
$$

The bound $\hat{\mathcal{E}}_{\text {loc }}$ is easy to implement (analytical computations may be possible) and the error on primal and adjoint solutions can be computed separately.

\subsection{Splitting of error sources}

In the problem we consider, the discretization error $I_{e x}-I_{h, m}$ on a given quantity of interest $I$ comes from two sources : (i) discretization of the space domain using a finite element mesh; (ii) discretization of the stochastic domain. In this section, we aim at assessing contributions of these two sources, in order to get relevant information that would help for driving adaptive procedures. The local error can be recast under the form :

$$
\mathcal{E}_{l o c}=\left[I_{e x}-I_{h}\right]+\left[I_{h}-I_{h, m}\right]=\mathcal{E}_{l o c, s p a}+\mathcal{E}_{l o c, s t o}
$$


where $I_{h}$ is the quantity of interest, corresponding to an exact resolution regarding randomness, but with a discretized solution using $\mathcal{M}_{h}$ regarding space. That way, $\mathcal{E}_{\text {loc,spa }}$ (resp. $\mathcal{E}_{\text {loc,sto }}$ ) is the contribution of the discretization error on $I$ due to the discretization of the space dimension (resp. stochastic dimension).

On the one hand, contribution $\mathcal{E}_{\text {loc,sto }}=I_{h}-I_{h, m}$ can be estimated using the goal-oriented error estimation method described previously, provided that the reference model which is considered is already discretized in space, i.e. taking the reference problem defined in Section 2.1 and applying a finite element discretization to it. With respect to this new reference problem, $I_{h}$ is the exact solution, and $I_{h, m}$ is an approximate solution obtained after discretization in the stochastic dimension.

In that framework, an admissible displacement/stress pair denoted $\left(\hat{\mathbf{u}}_{m}, \hat{\sigma}_{m}\right)$ shall be defined relative to this new reference model. In practice, such a pair can be automatically obtained as a simple postprocessing of the approximate solution $\left(\mathbf{u}_{h, m}, \sigma_{h, m}\right)$ at hand : we take $\hat{\mathbf{u}}_{m}=\mathbf{u}_{h, m}$, and construct $\hat{\sigma}_{m}$ as :

$$
\hat{\sigma}_{m}(\mathbf{x}, \theta)=\sum_{i=1}^{L} \sigma_{h}^{\chi_{i}}(\mathbf{x}) \cdot \chi_{i}(\theta)
$$

The construction of admissible fields $\left(\hat{\tilde{\mathbf{u}}}_{m}, \hat{\tilde{\sigma}}_{m}\right)$ for the new adjoint problem is similar. We eventually obtain the estimate $\hat{\mathcal{E}}_{\text {loc,sto }}=e_{c r e}\left(\hat{\mathbf{u}}_{m}, \hat{\sigma}_{m}\right) \cdot e_{c r e}\left(\hat{\tilde{\mathbf{u}}}_{m}, \hat{\tilde{\sigma}}_{m}\right)$, which is a guaranteed upper bound on the error $\left|I_{h}-I_{h, m}\right|$.

In the same way, contribution $\mathcal{E}_{l o c, s p a}=I_{e x}-I_{h} \approx I_{m}-I_{h, m}$ can be estimated taking as the reference model the one defined in Section 2.1 on which we apply the discretization in the stochastic dimension. With respect to this new reference problem, $I_{m}$ is the exact solution, and $I_{h, m}$ is an approximate solution obtained after discretization in the space dimension. An admissible displacement/stress pair denoted $\left(\hat{\mathbf{u}}_{h}, \hat{\sigma}_{h}\right)$, and relative to this new reference model, is again obtained as a simple post-processing of the approximate solution $\left(\mathbf{u}_{h, m}, \sigma_{h, m}\right)$ at hand : we take $\hat{\mathbf{u}}_{h}=\mathbf{u}_{h, m}$, and construct $\hat{\sigma}_{h}$ as : 


$$
\hat{\sigma}_{h}(\mathbf{x}, \theta)=\sum_{i=1}^{L} \hat{\sigma}_{h, m}^{i}(\mathbf{x}) \cdot \Psi_{i}(\theta)
$$

The construction of admissible fields $\left(\hat{\tilde{\mathbf{u}}}_{h}, \hat{\tilde{\sigma}}_{h}\right)$ for the new adjoint problem is similar. We eventually obtain the estimate $\hat{\mathcal{E}}_{l o c, s p a}=e_{c r e}\left(\hat{\mathbf{u}}_{h}, \hat{\sigma}_{h}\right) \cdot e_{\text {cre }}\left(\hat{\tilde{\mathbf{u}}}_{h}, \hat{\tilde{\sigma}}_{h}\right)$, which is a guaranteed upper bound on the error $\left|I_{m}-I_{h, m}\right|$.

\section{Numerical results}

\subsection{Test problems}

Two test-problems are considered here; the first (denoted $[A]$ ) is illustrated in Figure 2, the second (denoted $[B]$ ) is illustrated in Figure 3 . In both problems, Young's modulus $E_{1}$ is partially known in zone $\Omega_{1}$; we assume that this random variable (defined on $\Omega_{1}$ ) has a given probability density with mean $\overline{E_{1}}$ and variation $\delta_{1}$ :

$$
E_{1}(\theta)=\overline{E_{1}} \cdot\left[1+\delta_{1} g(\xi(\theta))\right] \quad \text { with } g(x)=\frac{2 \arcsin \left(\operatorname{Erf}\left(\frac{x}{\sqrt{2}}\right)\right)}{\sqrt{\pi^{2}-8}}
$$

where $\xi(\theta)$ is a Gaussian centered random variable. The nonlinear function $g$ is introduced, such that the probability density function $E_{1}(\theta)$ as a bounded support (this definition avoids negative Young's modulus values which would not be physically correct). The Young modulus $E_{2}$ is deterministic in zone $\Omega_{2}$.

On problem $[\mathrm{A}]$ the gamma shape structure is submitted to a given traction force $F_{d}^{x}$ along $\boldsymbol{x}$ axis and to a prescribed displacement $u_{d}^{y}$ along $\boldsymbol{y}$ axis and is clamped on the bottom boundary. On problem [B], the square structure is clamped on bottom and top boundaries, and submitted to prescribed displacement $u_{d}^{y}$ along $\boldsymbol{y}$ axis.

Data, loading, material, and geometry parameters are given in Table 1 for problems $[\mathrm{A}]$ and $[\mathrm{B}]$. 


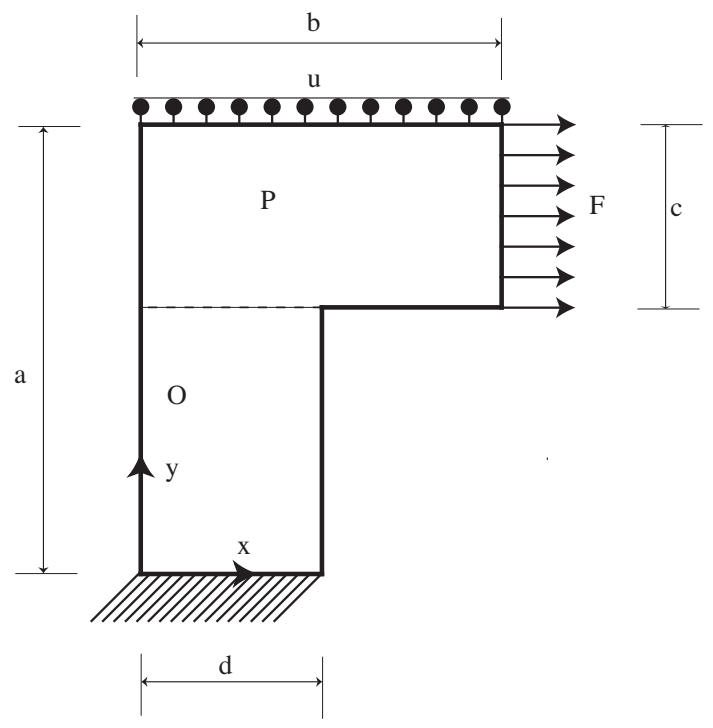

Figure 2. Definition of problem [A] : Gamma shape structure with clamped bottom boundary, prescribed displacement $u_{d}^{y}$ on top boundary, and prescribed traction $F_{d}^{x}$ on top-right boundary.

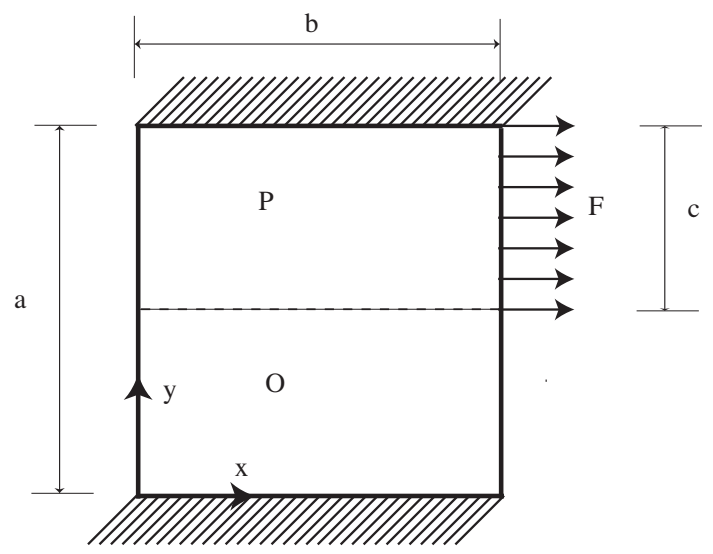

Figure 3. Definition of problem [B] : Square structure with clamped bottom and top boundaries, and prescribed traction $F_{d}^{x}$ on top-right boundary.

In both problems, the studied quantity of interest is the mean horizontal displacement on the application zone of $\mathbf{F}_{\mathbf{d}}$. More precisely :

$$
I=\int_{\Theta} \int_{\Omega} \tilde{\mathbf{f}}_{\Sigma} \cdot \mathbf{u} \mathrm{d} \Omega d P
$$

with : 
TABLE 1

\begin{tabular}{|c|c|c|c|c|c|c|c|c|c|}
\hline & $\overline{E_{1}}$ & $\delta_{1}$ & $E_{2}$ & $F_{d}^{x}$ & $u_{d}^{y}$ & $a$ & $b$ & $c$ & $d$ \\
\hline$[\mathrm{A}]$ & 1 & 0.1 & 2 & -1.5 & -2 & 25 & 20 & 10 & 10 \\
\hline$[\mathrm{B}]$ & 1 & 0.2 & 2 & 2 & - & 10 & 10 & 5 & - \\
\hline
\end{tabular}

Values of data, loading, material, and geometry parameters for problems $[\mathrm{A}]$ and $[\mathrm{B}]$.

$$
\tilde{\mathbf{f}}_{\Sigma}=\frac{1}{c} \delta_{x=b} \boldsymbol{x}
$$

$\delta_{x=b}$ being the classical Dirac function that localizes $I$ in the physical space.

\subsection{Bounding results}

On these simple test examples, the exact quantity of interest $I_{e x}$ is computed using an overkill solution, i.e. a very fine mesh $\left(10^{4}\right.$ quadratic elements) and a full Monte Carlo simulation $\left(10^{5}\right.$ samples $)$. Corresponding values are given in Table 2.

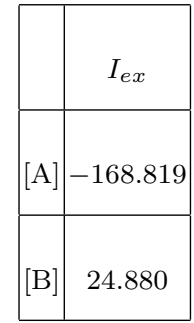

TABle 2

Value of $I_{e x}$ for problems $[\mathrm{A}]$ and $[\mathrm{B}]$.

An approximate solution is obtained performing a finite element computation, as explained in Section 2.2. Mesh $\mathcal{M}_{h}$ is uniform and constituted of first order quadrangular elements (see Figure 4). The scalar $h$ relative to $\mathcal{M}_{h}$ quality is defined as the number of finite elements on the edge $y=0$ of the structure. Grid $\mathcal{M}_{m}^{\prime}$ is constituted of piecewise linear elements, here the grid is $1 \mathrm{D}$. Parameter $m$, relative to the quality of $\mathcal{M}_{m}^{\prime}$ is the number of points on the $1 \mathrm{D}$-grid used to discretize $\Theta$.

A Von Mises stress repartition is illustrated in Figure 4 for problem [A]. 


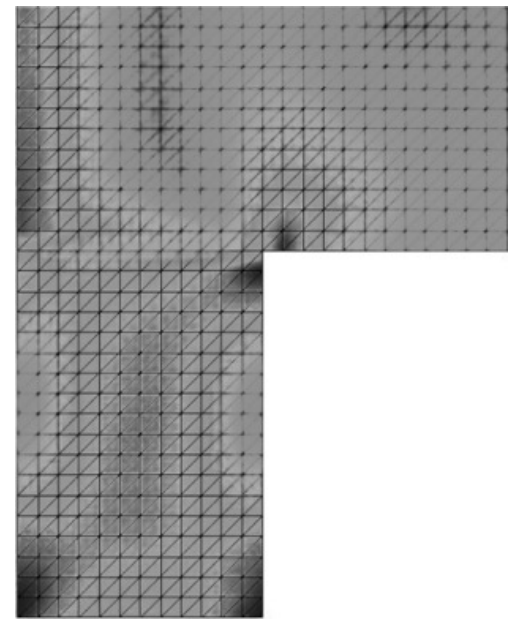

Figure 4. Finite element representation of a realization for the Von Mises equivalent stress.

For a given level of discretization $m$ and $h$, the value of the quantity $I_{h, m}$ is given in Table 3 .

TABLE 3

\begin{tabular}{|c|c|c|c|}
\hline & $m$ & $h$ & $I_{h, m}$ \\
\hline$[\mathrm{A}]$ & 11 & 12 & -185.591 \\
\hline$[\mathrm{B}]$ & 21 & 96 & 24.887 \\
\hline
\end{tabular}

Values of $I_{h, m}$ for problems $[\mathrm{A}]$ and $[\mathrm{B}]$.

Using the bounding technique (25) developed in Section 4, we obtain directly :

$$
I_{h, m}-\hat{\mathcal{E}}_{l o c} \leq I_{e x} \leq I_{h, m}+\hat{\mathcal{E}}_{l o c}
$$

and adimensional upper (resp. lower) bounds $\eta_{l o c}^{+}\left(\right.$resp. $\left.\eta_{l o c}^{-}\right)$may be defined in order to be compared to 1 , which gives an assessment of the quality of the bounds :

$$
\eta_{l o c}^{-}=\left(I_{h, m}-\hat{\mathcal{E}}_{l o c}\right) / I_{e x} \quad ; \quad \eta_{l o c}^{+}=\left(I_{h, m}+\hat{\mathcal{E}}_{l o c}\right) / I_{e x}
$$

The results are given in Table 4. 


\begin{tabular}{|c|c|c|c|c|c|}
\hline & $I_{h, m}-\hat{\mathcal{E}}_{l o c}$ & $I_{e x}$ & $I_{h, m}-\hat{\mathcal{E}}_{l o c}$ & $\eta_{l o c}^{-}$ & $\eta_{l o c}^{+}$ \\
\hline$[\mathrm{A}]$ & -207.863 & -168.819 & -163.319 & 0.9674 & 1.2313 \\
\hline$[\mathrm{B}]$ & 24.812 & 24.807 & 24.966 & 0.997 & 1.003 \\
\hline
\end{tabular}

TABLE 4

Bounds on $I_{e x}$ for problems $[\mathrm{A}]$ and $[\mathrm{B}]$.

\subsection{Refinement of the discretization}

In this section we present the evolution of the adimensional bounds with respect to the refinement of the space mesh (i.e. variation of parameter $h$ ), and the refinement of the grid (i.e. variation of parameter $m$ ). In Table 5, we give the different values of the adimensional bounds for different space mesh qualities $h, m$ being fixed, for problem [A]. In Table 6, we give the different values of the adimensional bounds for different mesh grid qualities $m, h$ being fixed, for problem [A].

\begin{tabular}{|c|c|c|c|c|c|}
\hline$m$ & $h$ & $I_{h, m}$ & $\hat{\mathcal{E}}_{l o c}$ & $\eta_{\text {loc }}^{-}$ & $\eta_{\text {loc }}^{+}$ \\
\hline 11 & 6 & -154.087 & 62.420 & 0.542 & 1.282 \\
\hline 11 & 12 & -163.188 & 22.275 & 0.834 & 1.098 \\
\hline 11 & 24 & -165.898 & 7.456 & 0.938 & 1.026 \\
\hline 11 & 48 & -166.997 & 2.554 & 0.974 & 1.004 \\
\hline 11 & 72 & -168.272 & 1.395 & 0.988 & 1.005 \\
\hline
\end{tabular}

TABLE 5

Evolution of the bounds with respect to the refinement of the space mesh size for problem [A].

In Table 7, we give the different values of the adimensional bounds for different space mesh qualities $h, m$ being fixed, for problem [B]. In Table 8, we give the different values of the adimensional bounds for different mesh grid qualities $m, h$ being fixed, for problem [B]. 
TABLE 6

\begin{tabular}{|c|c|c|c|c|c|}
\hline$m$ & $h$ & $I_{h, m}$ & $\hat{\mathcal{E}}_{l o c}$ & $\eta_{l o c}^{-}$ & $\eta_{l o c}^{+}$ \\
\hline 3 & 12 & -161.869 & 23.791 & 0.817 & 1.099 \\
\hline 5 & 12 & -162.911 & 22.527 & 0.831 & 1.098 \\
\hline 11 & 12 & -163.188 & 22.271 & 0.834 & 1.098 \\
\hline 21 & 12 & -162.714 & 22.218 & 0.832 & 1.095 \\
\hline 41 & 12 & -162.717 & 22.193 & 0.832 & 1.095 \\
\hline 81 & 12 & -162.718 & 22.180 & 0.832 & 1.095 \\
\hline
\end{tabular}

Evolution of the bounds with respect to the refinement of the grid size for problem [A].

\begin{tabular}{|l|c|c|c|c|c|}
\hline$m$ & $h$ & $I_{h, m}$ & $\hat{\mathcal{E}}_{l o c}$ & $\eta_{l o c}^{-}$ & $\eta_{l o c}^{+}$ \\
\hline 3 & 12 & 24.166 & 2.818 & 0.858 & 1.084 \\
\hline 3 & 24 & 24.668 & 0.963 & 0.952 & 1.030 \\
\hline 3 & 48 & 24.852 & 0.328 & 0.985 & 1.012 \\
\hline 3 & 96 & 24.921 & 0.130 & 0.996 & 1.006 \\
\hline 3 & 144 & 24.940 & 0.086 & 0.998 & 1.005 \\
\hline
\end{tabular}

TABLE 7

Evolution of the bounds with respect to the refinement of the space mesh size for problem [B].

\subsection{Estimation of contributions of various error sources}

We are now interested in the estimating parts of the error due to the stochastic (resp. space) discretization $\hat{\mathcal{E}}_{l o c, s t o}$ (resp. $\hat{\mathcal{E}}_{l o c, s p a}$ ) as explained in Section 4.3. For different values of $h$ and $m$, the results are given in Tables 9 and 10 (resp. Tables 11 and 12) for problem [A] (resp. problem [B]). 
TAble 8

\begin{tabular}{|l|l|l|l|l|l|}
\hline$m$ & $h$ & $I_{h, m}$ & $\hat{\mathcal{E}}_{l o c}$ & $\eta_{l o c}^{-}$ & $\eta_{l o c}^{+}$ \\
\hline 3 & 96 & 24.921 & 0.130 & 0.996 & 1.007 \\
\hline 5 & 96 & 24.895 & 0.084 & 0.997 & 1.004 \\
\hline 11 & 96 & 24.893 & 0.080 & 0.997 & 1.004 \\
\hline 21 & 96 & 24.887 & 0.079 & 0.997 & 1.003 \\
\hline 41 & 96 & 24.881 & 0.078 & 0.997 & 1.003 \\
\hline
\end{tabular}

Evolution of the bounds with respect to the refinement of the grid size for problem [B].

\section{Table 9}

\begin{tabular}{|c|c|c|c|c|}
\hline$m$ & $h$ & $\hat{\mathcal{E}}_{\text {loc,spa }}$ & $\hat{\mathcal{E}}_{\text {loc }, \text { sto }}$ & $\hat{\mathcal{E}}_{\text {loc }}$ \\
11 & 6 & 62.048 & $1,15 \mathrm{E}-04$ & 62.420 \\
\hline 11 & 12 & 22.285 & $1,21 \mathrm{E}-04$ & 22.275 \\
\hline 11 & 24 & 7.419 & $1,23 \mathrm{E}-04$ & 7.456 \\
\hline 11 & 48 & 2.525 & $1,22 \mathrm{E}-04$ & 2.554 \\
\hline
\end{tabular}

Evolution of the error contributions with respect to the refinement of the mesh size for problem [A].

Results on problem $[\mathrm{A}]$ show that the stochastic error is very low $\left(\hat{\mathcal{E}}_{\text {loc,sto }} \approx 1 \mathrm{E}-4\right.$ for $\left.m=11\right)$, and is negligible compared to the space error $\left(\hat{\mathcal{E}}_{l o c, s p a} \approx 22.2\right.$ for $\left.h=12\right)$.

On problem $[\mathrm{B}]$, the stochastic error $\left(\hat{\mathcal{E}}_{l o c, s t o} \approx 0.04\right.$ for $\left.m=3\right)$ is of the same order as the space error $\left(\hat{\mathcal{E}}_{\text {loc,spa }} \approx 0.085\right.$ for $\left.h=96\right)$.

Those error estimates may be useful if one wishes to adapt the discretization of the problem, as they give information on which dimension (space or stochastic) we should refine first. 


\begin{tabular}{|c|c|c|c|c|}
\hline$m$ & $h$ & $\hat{\mathcal{E}}_{\text {loc }, \text { spa }}$ & $\hat{\mathcal{E}}_{\text {loc }, \text { sto }}$ & $\hat{\mathcal{E}}_{\text {loc }}$ \\
3 & 12 & 22.181 & 0.174 & 23.791 \\
\hline 5 & 12 & 22.311 & 0.004 & 22.527 \\
\hline 11 & 12 & 22.285 & $1 \mathrm{E}-04$ & 22.271 \\
\hline 21 & 12 & 22.287 & $8 \mathrm{E}-06$ & 22.218 \\
\hline 41 & 12 & 22.287 & $4 \mathrm{E}-07$ & 22.193 \\
\hline 81 & 12 & 22.285 & $3 \mathrm{E}-08$ & 22.180 \\
\hline
\end{tabular}

TABLE 10

Evolution of the error contributions with respect to the refinement of the grid size [A].

\begin{tabular}{|l|l|l|l|l|}
\hline$m$ & $h$ & $\hat{\mathcal{E}}_{\text {loc }, \text { spa }}$ & $\hat{\mathcal{E}}_{\text {loc }, \text { sto }}$ & $\hat{\mathcal{E}}_{\text {loc }}$ \\
3 & 12 & 2.803 & 0.0418 & 2.818 \\
\hline 3 & 24 & 0.915 & 0.0424 & 0.963 \\
\hline 3 & 48 & 0.281 & 0.0426 & 0.328 \\
\hline 3 & 96 & 0.085 & 0.0428 & 0.130 \\
\hline 3 & 144 & 0.042 & 0.0428 & 0.086 \\
\hline
\end{tabular}

TABLE 11

Evolution of the error contributions with respect to the refinement of the mesh size for problem [B].

\section{Conclusions and prospects}

In this paper, we extended to the stochastic case the concept of goal-oriented error estimation based on the constitutive relation error. Considering linear elasticity problems, we showed how admissible fields could be constructed, and how they could be employed to build guaranteed error bounds on a given 
TABLE 12

\begin{tabular}{|l|l|l|l|l|}
\hline$m$ & $h$ & $\hat{\mathcal{E}}_{\text {loc }, \text { spa }}$ & $\hat{\mathcal{E}}_{\text {loc }, \text { sto }}$ & $\hat{\mathcal{E}}_{\text {loc }}$ \\
3 & 96 & 0.085 & 0.043 & 0.130 \\
\hline 5 & 96 & 0.086 & 0.003 & 0.084 \\
\hline 11 & 96 & 0.085 & $3.4 \mathrm{E}-04$ & 0.080 \\
\hline 21 & 96 & 0.085 & $2 \mathrm{E}-05$ & 0.079 \\
\hline 41 & 96 & 0.085 & $1 \mathrm{E}-06$ & 0.078 \\
\hline
\end{tabular}

Evolution of the error contributions with respect to the refinement of the grid size [B].

quantity of interest. We also proposed a simple procedure to assess separately contributions coming from various error sources (discretizations in space and stochastic dimensions in our case). The capabilities of these new tools were illustrated on 2D numerical experiments.

In future works, we wish to tackle problems with a large number of stochastic variables. We also wish to adapt to the stochastic case the non-intrusive procedure proposed in [6].

\section{References}

[1] Babuška I, Strouboulis T. The finite element method and its reliability. Oxford university press, 2001.

[2] Babuška I, Nobile F, Tempone R. A stochastic collocation method for elliptic partial differential equations with random input data. SIAM Journal on Numerical Analysis 2007; 45(3):1005-1034.

[3] Becker R, Rannacher R. An optimal control approach to shape a posteriori error estimation in finite element methods. A. Isereles (Ed.), Acta Numerica,Cambridge University Press, 2001; 10:1-120.

[4] Berveiller M. Stochastic finite elements: intrusive and non-intrusive methods for reliability analysis. PhD thesis, Université Blaise Pascal 2005.

[5] Chamoin L, Ladevèze P. Bounds on history-dependent or independent local quantities in viscoelasticity problems solved by approximate methods. International Journal for Numerical Methods in Engineering 2007; 71(12):1387-1411. 
[6] Chamoin L, Ladevèze P. A non-intrusive method for the calculation of strict and efficient bounds of calculated outputs of interest in linear viscoelasticity problems. Computer Methods in Applied Mechanics and Engineering 2008; 197(912):994-1014.

[7] Cirak F, Ramm E. A posteriori error estimation and adaptivity for linear elasticity using the reciprocal theorem. Computer Methods in Applied Mechanics and Engineering 1998; 156:351-362.

[8] Cottereau R, Diez P, Huerta A. Strict error bounds for linear solid mechanics problems using a subdomain-based flux-free method. Computational Mechanics 2009; 44(4):533-547.

[9] Deb M.K, Babuska I, Oden J.T. Solution of stochastic partial differential equations using Galerkin finite element techniques. Computer Methods in Applied Mechanics and Engineering 2001; 190:6359-6372.

[10] Florentin E, Gallimard L, Pelle J.P. Evaluation of the local quality of stresses in 3D finite element analysis. Computer Methods in Applied Mechanics and Engineering 2002; 191:4441-4457.

[11] Florentin E, Gallimard L, Pelle J.P, Rougeot Ph. Adaptive meshing for local quality of FE stresses. Engineering Computations 2005; 22:149-164.

[12] Florentin E, Ladevèze P, Bellec J. Error bounds on outputs of interest for linear stochastic problems. in Proceedings of the 3rd European Conference on Computational Mechanics 2006.

[13] Ghanem R, Spanos P.D. Stochastic Finite Elements: A Spectral Approach. Springer Verlag, 1991.

[14] Ghanem R, Pelissetti M. Error estimation for the validation of model-based predictions. in Proceedings of the 5th World Congress on Computational Mechanics 2002.

[15] Kolmogorov A.N. Foundations of the Theory of Probability. Chelsea Publishing Company, New York, 1956.

[16] Ladevèze P. Validation and verification of stochastic models in uncertain environment using constitutive relation error method. Technical Report 258, LMT-Cachan 2003.

[17] Ladevèze P. Strict upper error bounds for calculated outputs of interest in computational structural mechanics. Computational Mechanics 2008; 42(2):271-286.

[18] Ladevèze P, Pelle J-P. Mastering Calculations in Linear and Nonlinear Mechanics. Springer NY, 2004.

[19] Ladevèze P, Florentin E. Verification of stochastic models in uncertain environments using the constitutive relation error method. Computer Methods in Applied Mechanics and Engineering 2006; 196:225-234. 
[20] Ladevèze P, Chamoin L, Florentin E. A new non-intrusive technique for the construction of admissible stress fields in model verification. Computer Methods in Applied Mechanics and Engineering; 2010; 199(9-12):766-777.

[21] Ladevèze P, Waeytens J. Model verification in dynamics through strict upper error bounds. Computer Methods in Applied Mechanics and Engineering 2009; 198(21-26):1775-1784.

[22] Ladevèze P, Chamoin L. Calculation of strict error bounds for finite element approximations of non-linear pointwise quantities of interest. International Journal for Numerical Methods in Engineering; 2010; 84:1638-1664.

[23] Loeve M. Probability Theory (4 ${ }^{\text {th }}$ edition). Springer-Verlag, Berlin, 1977.

[24] Matthies H.G, Bucher C.G. Finite element for stochastic media problems. Computer Methods in Applied Mechanics and Engineering 1999; 168:3-17.

[25] Moitinho de Almeida J.P, Maunder E.A.W. Recovery of equilibrium on star patches using a partition of unity technique. International Journal for Numerical Methods in Engineering 2009; 79:1493-1516.

[26] Oden J.T, Babuška I, Nobile F, Feng Y, Tempone R. Theory and methodology for estimation and control of error due to modeling, approximation, and uncertainty. Computer Methods in Applied Mechanics and Engineering 2005; 194:195-204.

[27] Panetier J, Ladevèze P, Chamoin L. Strict and effective bounds in goal-oriented error estimation applied to fracture mechanics problems solved with XFEM. International Journal for Numerical Methods in Engineering 2010; 81:671-700.

[28] Paraschivoiu M, Peraire J, Patera A.T. A posteriori finite element bounds for linear functional outputs of elliptic partial differential equations. Computer Methods in Applied Mechanics and Engineering 1997; 150:289-312.

[29] Pares N, Diez P, Huerta A. Subdomain-based flux-free a posteriori error estimators. Computer Methods in Applied Mechanics and Engineering 2006; 195(4-6):297-323.

[30] Peraire J, Patera A.T. Bounds for linear-functional outputs of coercive partial differential equations; local indicators and adaptive refinements. Advances in Adaptive Computational Methods in Mechanics (Ladevèze and Oden Editors, Elsevier) $1998 ; 199-216$.

[31] Pled F, Chamoin L, Ladevèze P. On the techniques for constructing admissible stress fields in model verification: performances on engineering examples. International Journal for Numerical Methods in Engineering 2011; 88(5):409441. 
[32] Florentin, E., Guinard S., Pasquet, P. A simple estimator for stress errors dedicated to large elastic finite element simulations : Locally reinforced stress construction. Engineering Computations. 2011. 28:76-92

[33] Florentin E, Lubineau G. Identification of the parameters of an elastic material model using the Constitutive Equation Gap Method. Computational Mechanics 2010; 46:521-531.

[34] Prudhomme S, Oden J.T. On goal-oriented error estimation for elliptic problems: application to the control of pointwise errors. Computer Methods in Applied Mechanics and Engineering 1999; 176:313-331.

[35] Romkes A, Oden J.T, Vemaganti K. Multiscale goal-oriented adaptive modeling of random heterogeneous materials. Mechanics of Materials 2006; 38:859-872.

[36] Schueller G. A state-of-the-art report on computational stochastic mechanics. Probabilistic Engineering Mechanics $1997 ; 12: 197-321$.

[37] Sudret B, Berveiller M, Lemaire M. Eléments finis stochastiques en élasticité linéaire. Comptes Rendus de l'Académie des Sciences, Mécanique 2004; 332:531-537.

[38] Stein E, Ohmnimus S, Walhorn E. Local error estimates of fem for displacements and stresses in linear elasticity by solving local Neumann problems. International Journal for Numerical Methods in Engineering 2001; 52:727-746.

[39] Stefanou G. The stochastic finite element method: Past, present and future. Computer Methods in Applied Mechanics and Engineering 2009; 198:1031-1051. 